\title{
Evolution of the Growth Front for Copper Electrodeposition Followed by Real Time Imaging
}

\author{
P. Schilardi, S. Méndez, R. C. Salvarezza, and A. J. Arvia* \\ Instituto de Investigaciones Fisicoquímica Teóricas y Aplicadas (INIFTA), \\ Facultad de Ciencias Exactas, Universidad Nacional de La Plata-CONICET, \\ Sucursal 4, Casilla de Correo 16, (1900) La Plata, Argentina
}

Received December 10, 1997. In Final Form: March 23, 1998

\begin{abstract}
The interface motion for $\mathrm{Cu}$ electrodeposition in additive-free and thiourea-containing acid plating baths using three-dimensional and quasi-two-dimensional electrochemical cells was followed in real time in the potential range where the kinetics of the reaction is dominated by activation and Ohmic overpotentials. In the additive-free plating bath the growing interface changes from a marginally stable (nodular) to an unstable (branched) regime as the activation overpotential/Ohmic overpotential ratio decreases. The presence of thiourea drives the interface motion to a steady-state regime in which the predictions of the Edwards-Wilkinson equation for the interface motion are obeyed. The influence of thiourea on $\mathrm{Cu}$ electrodeposition is to slow the growth rate at protrusions and to increase the growth rate at flat surface domains or valleys, leading to a smoothing of the entire growing electrodeposit surface.
\end{abstract}

\section{Introduction}

The dynamics of solid surfaces is an active area of experimental and theoretical research ${ }^{1}$ that includes a number of different situations such as a change in the type and density of surface defects at single-crystal surfaces, a displacement of adsorbates on the surface, and changes induced by external forces. Knowledge of these phenomena including their kinetics and mechanisms is required to improve the quality of metal films prepared by vapor deposition, molecular beam epitaxy, chemical vapor deposition, and electrodeposition.

Metal electrodeposition is widely used to prepare films with specific characteristics by properly adjusting the growth rate conditions through the applied electric potential, the composition of the plating solution, and temperature. In this way growth patterns ranging from a self-similar fractal to a rough compact pattern can be obtained. ${ }^{2}$ While the origin of branched morphologies at high current densities can be understood in terms of diffusion-limited aggregation models, ${ }^{2}$ the origin of branched deposits at low current densities in the absence of mass transport control is still a matter of discussion. ${ }^{3}$ In fact, unstable growth fronts at low deposition rates have been tentatively explained by considering either the influence of the electric field ${ }^{4}$ or the occurrence of an energy barrier at step edges ${ }^{5}$ or shadowing effects among growing crystals. ${ }^{1}$ Therefore, the origin of the unstable growth regime is an open question which deserves further work.

The smoothness and brightness of $\mathrm{Ni}, \mathrm{Zn}, \mathrm{Sn}$, and $\mathrm{Cu}$ electrodeposits can be substantially improved by adding

\footnotetext{
* To whom correspondence should be addressed.
}

(1) Stanley, E.; Barabasi, A. Fractal Concepts in Surface Growth; Cambridge University Press: New York, 1995; and references therein.

(2) Matshushita, M. In The Fractal Approach to the Heterogeneous Chemistry, Avnir, D., Ed.; J. Wiley: New York, 1989; p 161. Meakin, P. Phys. Rep. 1993, 235, 191 and references therein.

(3) Kahanda, G. L. M. K. S.; Zou, X.; Farrell, R.; Wong, P. Phys. Rev. Lett. 1992, 68, 3741. Pastor, J. M.; Rubio, M. A. Phys. Rev. Lett. 1996 $76,1848$.

(4) Iwamoto, A.; Yoshinobu, T.; Iwasaki, H. Phys. Rev. Lett. 1994, 72 4025 .

(5) Schwoebel, R. L. J. Appl. Phys. 1968, 40, 614. Zhang, Z.; Deutch, J.; Matiu, H. Phys. Rev. B 1993, 48, 3806. to the plating bath certain organic compounds (additives) in small amounts. ${ }^{6}$ In principle, additive molecules in plating baths can be either adsorbed or electroadsorbed on the surface of the growing phase, and a small amount of additive can also be incorporated into the growing phase itself. Nevertheless, despite the intensive practical use of additives in metal plating baths, neither a comprehensive explanation nor a convincing physicochemical theory is still available for the role of additives in the growth mode of metal electrodeposits. ${ }^{7}$

A great effort has been made to describe the evolution of growing interfaces by means of continuous equations (continuous models). ${ }^{1}$ Starting from a planar substrate, the interface motion has been described by partial differential equations involving competing contributions from the stochastic noise of depositing particles and surface relaxation phenomena. ${ }^{1}$ But at present the agreement between modeling and experimental data is, in general, far from satisfactory. ${ }^{8}$ In contrast to this situation, it has recently been found that, for $\mathrm{Cu}$ electrodeposits produced from a plating bath containing a thiourea derivative ${ }^{9}$ the variation with time of the interface height resulting from the analysis of ex-situ atomic force microscopy (AFM) images appears to obey the simple equation ${ }^{1,10}$

$$
\partial h(\mathbf{x}, t) / \partial t=v \nabla^{2} h-K \nabla^{4} h+\eta(\mathbf{x}, t)
$$

where $h(\mathbf{x}, t)$ is the interface height at any position $\mathbf{x}$ and time $t, v$ is the surface tension coefficient, and $K$ is a constant. The first linear term on the right hand side of eq 1 corresponds to the surface relaxation contribution operating through adsorption/desorption-type processes, ${ }^{11}$

(6) Despic, A. R. In Comprehensive Treatise of Electrochemistry Conway, B. E., Bockris, J. O’M., Yaeger, E., Khan, S. U. M., White, R E., Eds.; Plenum Press: New York, 1983; Vol. 7, p 451.

(7) Barkey, D. P.; Muller, R. H.; Tobias, C. W. J. Electrochem. Soc 1989, 136, 2207.

(8) Krim, J.; Palasantzas, G. Int. J. Mod. Phys. B 1995, 9, 599.

(9) Mendez, S.; Andreasen, G.; Schilardi, P.; Figueroa, M.; Vázquez L.; Salvarezza, R. C.; Arvia, A. J. Langmuir 1998, 14, 2515.

(10) Wolf, D.; Villain, J. Europhys. Lett. 1990, 13, 389. Villain, J. J. Phys. I 1992, 1, 19.

(11) Edwards, S. F.; Wilkinson, D. R. Proc. R. Soc. London A 1982 381,17 . 
and the second linear term refers to surface relaxation through surface diffusion. ${ }^{10}$ The term $\eta(\mathbf{x}, t)$ in eq 1 stands for the white Gaussian noise related to the aggregation of finite-sized particles. The competition between linear terms generates a characteristic horizontal length scale defined by $L_{\mathrm{c}}=(K / v)^{1 / 2}$, so that for a scaling length $L<$ $L_{\mathrm{c}}$ the interface evolution is dominated by the second term of eq 1, leading to the Villain-Wolf rate equation, ${ }^{10}$ whereas for $L>L_{\mathrm{c}}$ the first term in eq 1 determines the scaling behavior of the system, leading to the EdwardsWilkinson $(\mathrm{EW})$ rate equation. ${ }^{11}$

The fact that a complex system such as that involved in $\mathrm{Cu}$ electrodeposition can be described on the asymptotic limit by a relatively simple equation such as the EW equation is, in principle, unexpected. For this reason, the EW behavior of the electrodepositing $\mathrm{Cu}$ interface must be confirmed by extending the dynamic scaling analysis to the evolution of relevant topographic features in real time covering a range of $L$ larger than that previously accessible by AFM imaging data.

This work deals with the real time interface motion of both the electrodepositing $\mathrm{Cu}$ /additive-free and the electrodepositing $\mathrm{Cu}$ /thiourea-containing acid plating bath interfaces. In the former case, the growing interface changes from a marginally stable (nodular) regime to an unstable (branched) regime as the activation overpotential/Ohmic overpotential ratio decreases. This ratio determines the degree of uniformity for the current distribution over the entire growing electrodeposit.

The presence of the additive in the plating bath drives the interface from an unstable to a stable roughness regime. From the motion of instabilities in real time, the fulfillment of the EW model earlier concluded from the analysis of ex-situ AFM imaging data ${ }^{9}$ is confirmed and extended to larger values of $L$. The analysis of combined interface motion and electrochemical data furnishes for the first time a physicochemical picture of the role played by the presence of additives in $\mathrm{Cu}$ electrodeposition, which supports the mathematical model.

\section{Experimental Section}

Copper electrodeposits were grown under a constant apparent current density, $j=0.02 \mathrm{~A} \mathrm{~cm}^{-2}$, at $298 \mathrm{~K}$, from aqueous solutions with the composition $0.6 \mathrm{M} \mathrm{CuSO}_{4} \cdot 5 \mathrm{H}_{2} \mathrm{O}+0.5 \mathrm{M} \mathrm{H}_{2} \mathrm{SO}_{4}+x(0$ $\leq x \leq 0.3) \mathrm{mM}$ thiourea. Plating solutions were prepared from a.r. quality chemicals and MilliQ-Millipore water and stored under purified $\mathrm{N}_{2}$ saturation for $2 \mathrm{~h}$ before each electrochemical run.

Polarization curves were run using either a conventional threedimensional electrochemical glass cell (hereafter denoted as a $3 \mathrm{D}$ cell) or quasi-two-dimensional rectangular electrochemical glass cells (hereafter denoted as quasi-2D cells), at the potential scan rate $v=10^{-2} \mathrm{~V} \mathrm{~s}^{-1}$. This value of $v$ represents a compromise between a reasonable approach to quasi-steady-state electrodeposition conditions and a small change in the surface area of $\mathrm{Cu}$ electrodeposits. For the 3D cell, a Cu disk $\left(0.196 \mathrm{~cm}^{2}\right.$ geometric area) was utilized as working cathode, and a $\mathrm{Cu}$ reference electrode was placed very near to the cathode either to compensate for or to minimize the Ohmic drop correction, depending whether a 3D or a quasi-2D cell was employed, respectively. In both cells, a $\mathrm{Cu}$ anode of a geometric area larger than that of the cathode was used.

The quasi-2D cells (Figure 1) consisted of a parallel electrode arrangement of a Cu cathode $\left(0.038 \mathrm{~cm}^{2} \leq\right.$ geometric area $\leq 0.13$ $\left.\mathrm{cm}^{2}\right)$ and a $\mathrm{Cu}$ anode $\left(4.0 \mathrm{~cm}^{2}\right.$ geometric area) made from a 0.05 $\mathrm{cm}$-thick Cu strip (99.99\% purity). The anode-to-cathode distance in these cells was $d_{\mathrm{ac}}=1.3 \mathrm{~cm}$. To determine the influence of a characteristic length on the morphology of the electrodeposits, the value of $r$, the anode to cathode geometric area ratio, was varied in the range $37 \leq r \leq 200$.

Conventional cathodic polarization curves run with the different plating solutions were plotted as either the cathodic

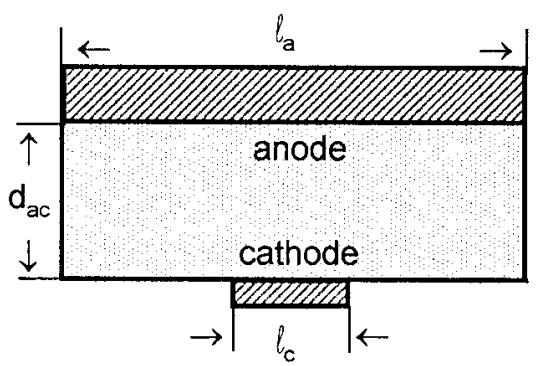

Figure 1. Scheme of an ideal 2D rectangular cell. $d_{\mathrm{ac}}$ is the anode-to-cathode distance; $l_{\mathrm{c}}$ is the cathode length; $l_{\mathrm{a}}$ is the anode length.

overvoltage $\left(\Delta E_{\mathrm{c}}\right)$ versus $j$ or the $\Delta E_{\mathrm{c}}$ versus $\log j$ plot, where $\Delta E_{\mathrm{c}}$ $=E_{\mathrm{rev}}\left(\mathrm{Cu} / \mathrm{Cu}^{2+}\right)-E$ with $E_{\mathrm{rev}}\left(\mathrm{Cu} / \mathrm{Cu}^{2+}\right)$ the initial reversible potential of the $\mathrm{Cu}$ electrode in the solution and $E$ the electric potential applied to the cathode.

The evolution of the topography of each Cu electrodeposit was followed at $298 \mathrm{~K}$ using a videomicroscopy imaging system with a 750 pixel $\times 570$ pixel resolution. Images were taken in the range $0.8 \mathrm{~mm} \leq L \leq 1.30 \mathrm{~mm}$. Since there is a critical thiourea concentration above which smoothing is produced, the initial thiourea concentration was adjusted within a range in which the fluctuations of the interface width were in the micrometer to millimeter range. The reproducibility of morphology was verified from at least triplicate runs.

\section{Results and Interpretation}

3.1. Polarization Curves. Data from the polarization curves depend both on the concentration of the additive in the plating bath and on the geometry of the electrochemical cell.

In the $3 \mathrm{D}$ cell, the cathodic polarization curve for $\mathrm{Cu}$ electrodeposition on $\mathrm{Cu}$ from the additive-free plating bath, that is, $x=0$ (Figure 2a), exhibits two well-defined regions. The first region covers the potential range $0 \leq \Delta E_{\mathrm{c}} \leq 0.2$ $\mathrm{V}$. This region is practically Ohmic polarization-free and fits a $\Delta E_{\mathrm{c}}$ versus $\log j$ linear Tafel relationship (Figure $2 \mathrm{~b}$ ) with the slope $0.12 \pm 0.005 \mathrm{~V} /$ decade. This slope agrees with the ratio $2.303(2 R T / F)$ at $298 \mathrm{~K}$, where $R$ and $F$ are the universal gas constant and the Faraday constant, respectively. The extrapolation of the straight line to $\Delta E_{\mathrm{c}}$ $=0$ yields an apparent exchange current density $j_{0} \cong 0.8$ $\times 10^{-3} \mathrm{~A} \mathrm{~cm}^{-2}$, as already reported for a similar plating bath. ${ }^{12-14}$ Thus, in the range $0 \leq \Delta E_{\mathrm{c}} \leq 0.2 \mathrm{~V}$ both the Tafel slope and the value of $j_{0}$ are consistent with those expected for an electrochemical reaction dominated by activation control, as already reported. ${ }^{15}$ In fact, for $j=$ $0.02 \mathrm{~A} \mathrm{~cm}^{-2}$, the contribution of the concentration overpotential $\eta_{\mathrm{c}}$ is less than $10 \%$ of the value of $\Delta E_{\mathrm{c}}{ }^{9}{ }$ Therefore, in this case, it can be admitted that $\Delta E_{\mathrm{c}} \cong \eta_{\mathrm{a}}$, where $\eta_{\mathrm{a}}$ stands for the activation overpotential, as concluded from the characteristics of the Tafel plot. Otherwise, for $\Delta E_{\mathrm{c}}$ $>0.20 \mathrm{~V}$, the cathodic current first reaches a maximum value that depends on $v$ and then attains a limiting current that depends linearly on the concentration of $\mathrm{Cu}^{2+}$ ions in the solution, in agreement with an electrochemical reaction which, for $\Delta E_{\mathrm{c}}>0.5 \mathrm{~V}$, is definitely under steady mass transport rate control, ${ }^{13}$ and in this case $\Delta E_{\mathrm{c}} \cong \eta_{\mathrm{c}}$

(12) Bertocci, U.; Turner, D. In Encyclopedia of Electrochemistry of the Elements; Bard, A. J., Ed.; M. Dekker: New York, 1976; Vol. 2, p 383.

(13) Hine, F. Electrode Processes and Electrochemical Engineering, Plenum Press: New York, 1985

(14) Smyrl, W. H. In Comprehensive Treatise of Electrochemistry; Bockris, J. O’M., Conway, B. E., Yaeger, E., White, R. E., Eds.; Plenum Press: New York, 1981; Vol. 4, p. 97.

(15) Bockris, J. O’M.; Reddy, A. K. N. Modern Electrochemistry Macdonald: London, 1970; Vol. 2, p 1202. 
a

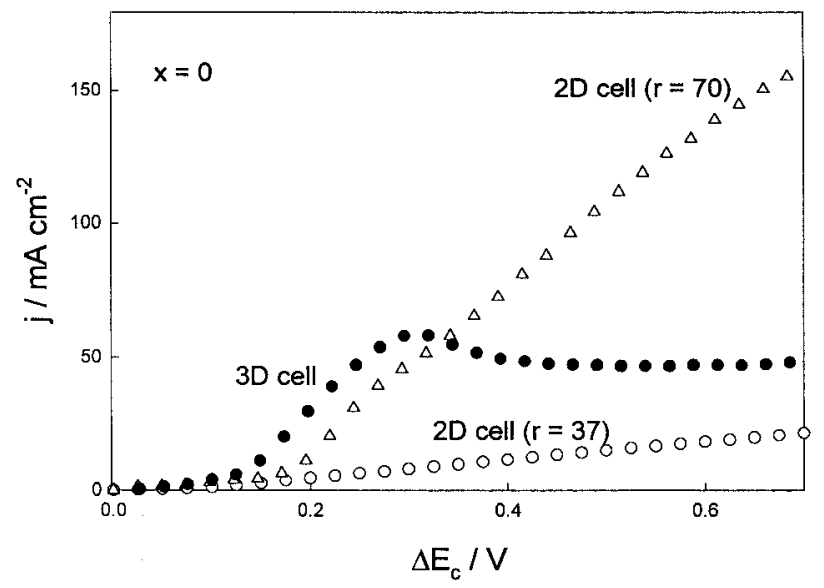

b

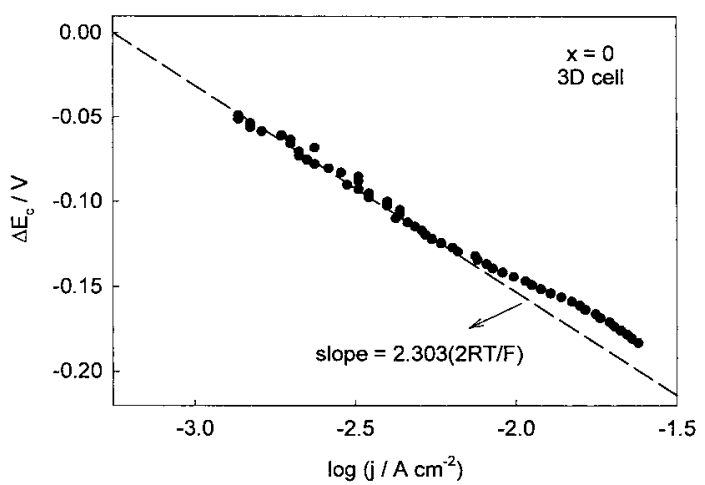

Figure 2. (a) $\Delta E_{\mathrm{c}}$ versus $j$ plots for $\mathrm{Cu}$ electrodeposition from $0.6 \mathrm{M} \mathrm{CuSO}_{4} \cdot 5 \mathrm{H}_{2} \mathrm{O}+0.5 \mathrm{M} \mathrm{H}_{2} \mathrm{SO}_{4}$ at $298 \mathrm{~K}$, using the $3 \mathrm{D}$ and 2D $(r=37$ and $r=70)$ cells. (b) $\Delta E_{\mathrm{c}}$ versus $\log j$ plots for $\mathrm{Cu}$ electrodeposition from $0.6 \mathrm{M} \mathrm{CuSO}_{4} \cdot 5 \mathrm{H}_{2} \mathrm{O}+0.5 \mathrm{M} \mathrm{H}_{2} \mathrm{SO}_{4}$ at $298 \mathrm{~K}$, using the 3D cell. Data from the initial portion of the polarization curve shown in (a) are plotted. The theoretical Tafel line corresponding to the slope $0.118 \mathrm{~V} /$ decade and $j_{0}=$ $0.8 \times 10^{-3} \mathrm{~A} \mathrm{~cm}^{-2}$ is drawn.

Similar runs were made in quasi-2D cells involving a parallel electrode design with $r=37$ and $r=200$. In these cases, the polarization curves (Figure 2a) are substantially different from those described above. Thus, for $r=37$, the $\Delta E_{\mathrm{c}}$ versus $j$ plot shows a tendency to approach an initial exponential current density increase followed by a highly distorted limiting current density in the range $0.4 \mathrm{~V} \leq \Delta E_{\mathrm{c}} \leq 0.6 \mathrm{~V}$. These features indicate a substantial Ohmic overpotential $\left(\eta_{0}\right)$ contribution in the polarization curve, and therefore, in this case $\Delta E_{\mathrm{c}} \cong \eta_{\mathrm{a}}+$ $\eta_{\mathrm{o}}$. The relative contribution of $\eta_{0}$ decreases with $r$. Thus, for $r=70$ the $\Delta E_{\mathrm{c}}$ versus $j$ plot approaches Ohm law with the slope predicted from the specific conductance of the plating bath $\left(\kappa=0.160 \Omega^{-1} \mathrm{~cm}^{-1}\right)$ and the geometry of the cell.

Considering that the value of $\eta_{\mathrm{a}}$ for $j=0.02 \mathrm{~A} \mathrm{~cm}^{-2}$ resulting from runs made in the $3 \mathrm{D}$ cell (Figure $2 \mathrm{a}$ ) is $\eta_{\mathrm{a}}$ $=0.12 \mathrm{~V}$ and taking into account the values of $\Delta E_{\mathrm{c}}$ for $j$ $=0.02 \mathrm{~A} \mathrm{~cm}^{-2}$ obtained from runs carried out in $2 \mathrm{D}$ cells, it follows that $\eta_{0}=0.1 \mathrm{~V}$ and $\eta_{0}=0.3 \mathrm{~V}$ for $r=70$ and $r=37$, respectively. Then, these values of $\eta_{\mathrm{a}}$ and $\eta_{0}$ indicate that a mixed kinetics involving mainly activation and Ohmic overpotentials determines the growth mode of $\mathrm{Cu}$ electrodeposits from the additive-free electroplating bath at quasi-2D cells.

Cathodic polarization curves for $\mathrm{Cu}$ electrodeposition from thiourea-containing plating baths in the 3D cell (Figure 3a) in the range $0<\Delta E_{\mathrm{c}}<0.3 \mathrm{~V}$ are drastically
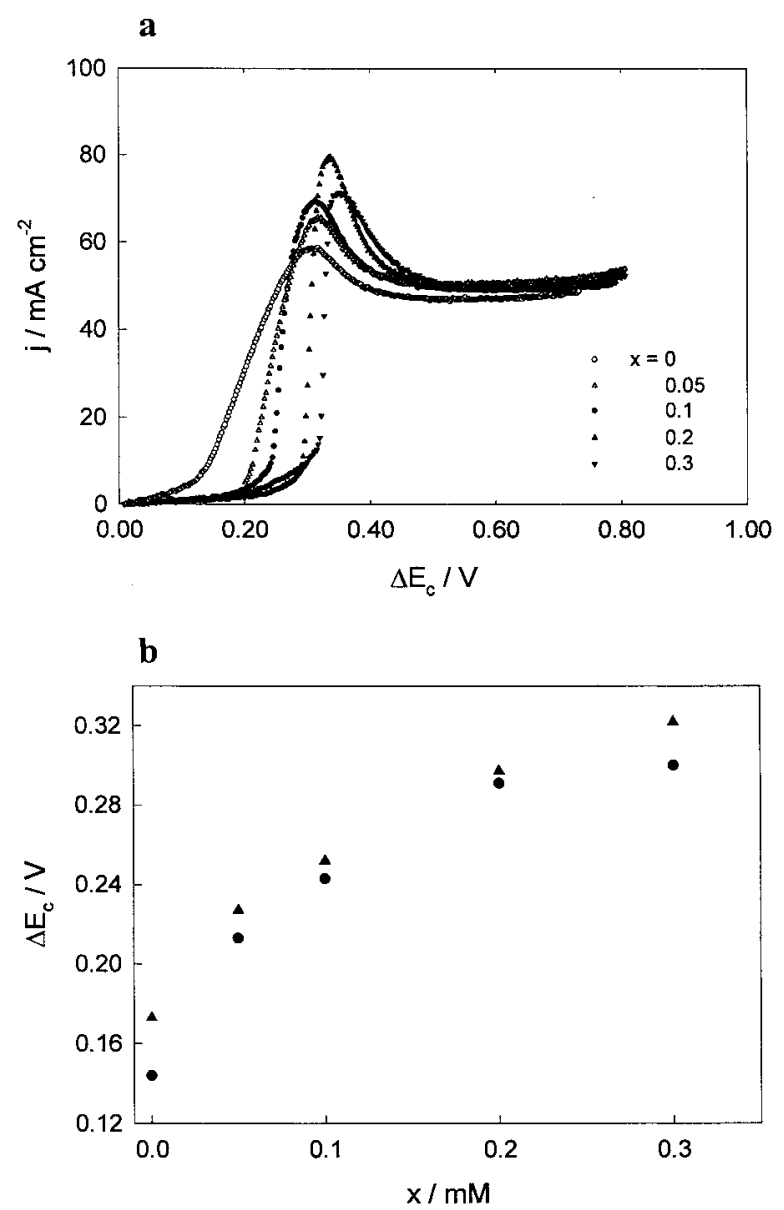

Figure 3. (a) $\Delta E_{\mathrm{c}}$ versus $j$ plots for $\mathrm{Cu}$ electrodeposition from plating baths with different values of $x$ using the 3D cells, 298 K. (b) $\left(\Delta E_{\mathrm{c}}\right)_{\mathrm{j}}$ versus $x$ plots for $j=0.01 \mathrm{~A} \mathrm{~cm}^{-2}(\bullet)$ and $j=0.02$ $\mathrm{A} \mathrm{cm}-2(\boldsymbol{\Lambda})$. Data taken from part (a).

different from those resulting from the thiourea-free bath. This difference becomes more remarkable as $x$ is increased (Figure $3 a$ ); that is, the value of $\Delta E_{\mathrm{c}}$ required for attaining a steady value of $j$ increases with $x$ (Figure 3b). These results show that the major influence of thiourea appears as an additional resistance to the electrochemical reaction at surface sites rather than as a mass transport resistance from the solution side. This conclusion is supported by the common value of the cathodic limiting current density that is approached, irrespective of $x$.

To discriminate whether the additional resistance produced by the presence of thiourea operates either locally or uniformly over the entire growing metal surface, a further analysis of polarization curves was made. Under equilibrium conditions, it is known that the degree of surface coverage by thiourea $\left(\theta_{\mathrm{e}}\right)$ increases with $x$ according to a Frumkin-type adsorption isotherm. ${ }^{16}$ In our experiments, the system should be considered under nonequilibrium adsorption conditions because of the rapid renewal of the surface due to the $\mathrm{Cu}$ electrodeposition rate at $j=$ $0.02 \mathrm{~A} \mathrm{~cm}^{-2}\left(18 \mathrm{~nm} \mathrm{~s}^{-1}\right)$, which implies an average halflife time for a monolayer of electrodeposited $\mathrm{Cu}$ of less than $0.01 \mathrm{~s}$. As this time is much smaller than that required to attain adsorption equilibrium over the entire interface, the average value of $\theta$ is $\langle\theta\rangle\left\langle\theta_{\mathrm{e}}\right.$. Then, for $j$ $=0.02 \mathrm{~A} \mathrm{~cm}^{-2}$ the greater values of $\Delta E_{\mathrm{c}}$ with increasing $x$ and the tendency to attain a potential plateau (Figure

(16) Holze, R.; Schomaker, S. Electrochim. Acta 1990, 35, 613. Holzle, M. H.; Apsel, C. W.; Will, T.; Kolb, D. M. J. Electrochem. Soc. 1995, 142, 3741 . 
a
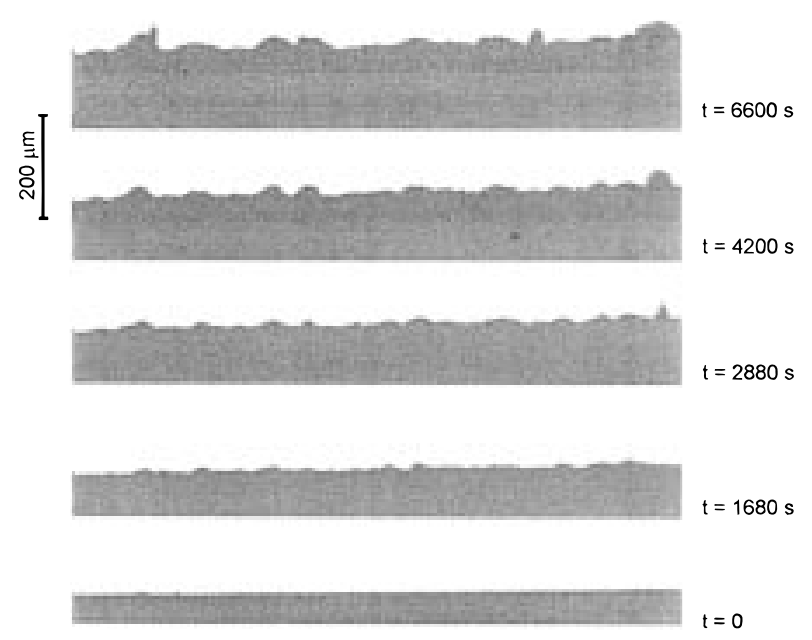

b

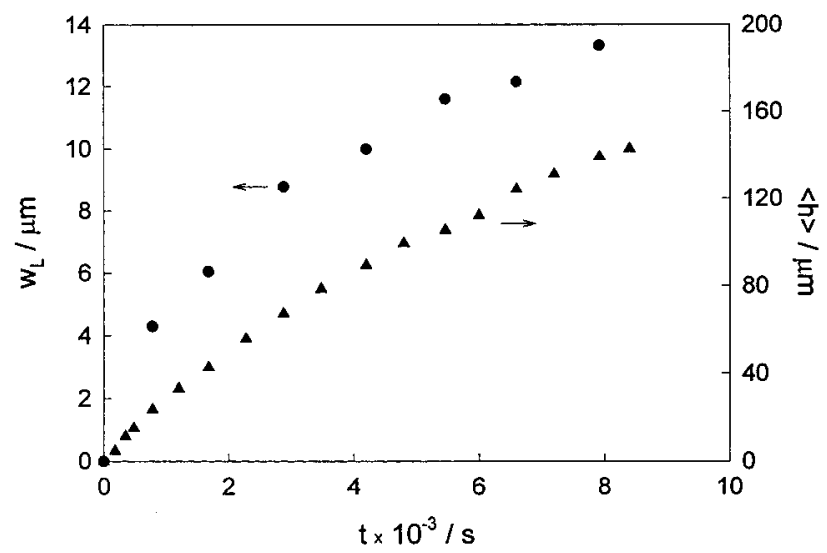

Figure 4. (a) In-situ micrographs showing the interface evolution for $\mathrm{Cu}$ electrodeposition from $0.6 \mathrm{M} \mathrm{CuSO}_{4} \cdot 5 \mathrm{H}_{2} \mathrm{O}+$ $0.5 \mathrm{M} \mathrm{H}_{2} \mathrm{SO}_{4}$ at $298 \mathrm{~K}$, using the $2 \mathrm{D}(r=90)$ cell. Gray shadowed portions correspond to the $\mathrm{Cu}$ phase. (b) $w_{\mathrm{L}}$ versus $t$ and $\langle h\rangle$ versus $t$ plots resulting from data taken from in-situ micrographs. The experimental conditions are the same as those for part (a).

$3 \mathrm{~b})$ should be related to an increase in the degree of surface coverage for $x<0.025$ and to a maximum degree of surface coverage $(\theta)$ by thiourea attained for $x>0.025$. This means that at the highest steady electrodeposition rates (limiting current region) the value of $\langle\theta\rangle$ would approach zero $\left(\langle\theta\rangle \ll \theta_{\mathrm{e}}\right)$, leading to an almost $x$-independent cathodic limiting current density at high values of $\Delta E_{\mathrm{c}}$ (Figure 3a).

3.2. Lateral Imaging of Growing Electrodeposits. The sequential imaging of $\mathrm{Cu}$ electrodeposits produced from a thiourea-free plating bath for $j=0.02 \mathrm{~A} \mathrm{~cm}^{-2}$ and $r=90$ (Figure 4a) shows the formation of large nodular structures, the average size of nodules increasing with time. The nodular patterns could be followed for at least $12 \mathrm{~h}$ of Cu electrodeposition. Similar patterns are obtained in those experiments involving the same electrodeposition charge for $r=90$ and adjusting $j=0.001 \mathrm{~A} \mathrm{~cm}^{-2}$.

The average height $(\langle h\rangle)$ of the deposit fits a $\langle h\rangle \propto t^{\mathrm{P}}$ relationship, whereas the root-mean-square roughness (interface width) $\left(w_{\mathrm{L}}\right)$ fulfills a $w_{\mathrm{L}} \propto t^{\beta}$ relationship. From these proportionalities $p=\beta=0.6 \pm 0.08$ (Figure 4b), a figure which agrees with that derived from ex-situ AFM imaging data on $\mathrm{Cu}$ electrodeposits grown in 3D cells. ${ }^{9}$ The coincidence of results from the 3D and quasi-2D cells in which different measuring directions of the deposit are considered indicates that $\mathrm{Cu}$ electrodeposit growing fronts produced in quasi-2D cells actually behave as 3D objects, since the maximum value of $w_{\mathrm{L}}$ in Figure $4 \mathrm{~b}$ is remarkably smaller than $0.05 \mathrm{~cm}$, the quasi-2D cell thickness. But, it is most important that the same $\beta$ value is derived from experiments at different scale lengths, such as optical microscopy and AFM. ${ }^{9}$

The analysis of the interface evolution (Figure 4a) clearly shows that the phase growth is dominated by the motion of large nodules capturing most of the depositing $\mathrm{Cu}$ ions, whereas valleys among nodules capture only a small amount of $\mathrm{Cu}$ ions and the electrodeposit growth rate there is lower. Consequently, the interface width increases continuously without attaining saturation. Then, the dynamic scaling approach breaks down and $\beta$ should be considered as an effective exponent ${ }^{1}$ that changes from 0.7 to 1 depending whether its value results from the $w_{\mathrm{L}}$ versus $t$ or the $w_{\mathrm{L}}$ versus $\langle h\rangle$ plot. Clearly, the small slope theory approximation ${ }^{1}$ for interface growth such as that involved in eq 1 is not applicable to Cu electrodeposition at $j=0.02 \mathrm{~A} \mathrm{~cm}^{-2}$.

On the other hand, the images of $\mathrm{Cu}$ electrodeposits resulting from $r=37$ (Figure 5a) show deep valleys separating nodules and the tendency to nodule splitting. Both the $\langle h\rangle$ versus $t$ and the $w_{\mathrm{L}}$ versus $t$ plots resulting from these experiments follow a power-like dependence with an exponent lower than 1 for $t<2.5 \times 10^{3} \mathrm{~s}$ and an exponential increase for $t>2.5 \times 10^{3} \mathrm{~s}$ (Figure $5 \mathrm{~b}$ ).

The different deposit morphologies resulting from $j=$ $0.02 \mathrm{~A} \mathrm{~cm}^{-2}$ using quasi-2D cells with different values of $r$ can be explained considering Wagner's number, which through the decrease in the $\eta_{\mathrm{a}} / \eta_{0}$ ratio reflects the departure of the system from a uniform current distribution. In fact, for $j=0.02 \mathrm{~A} \mathrm{~cm}^{-2}\left(\eta_{\mathrm{a}}=0.12 \mathrm{~V}\right)$, as $r$ increases from 37 to 200, $\eta_{0}$ increases from 0.1 to $0.3 \mathrm{~V}$, resulting in a less uniform current distribution at the cathode. This effect drives the interface evolution from a marginal stable regime (nodular) to an unstable regime (branching).

Marginal stable or unstable growth fronts have been assigned to different contributions, namely, (i) the presence of a Laplacian field assisting Cu growth at protrusions normal to the deposit surface, ${ }^{3}$ (ii) a shadowing effect among growing crystals, ${ }^{1}$ (iii) the presence of a spatial or correlated noise, ${ }^{17-18}$ and (iv) the existence of an energy barrier at step edges (Schwoebel barrier) hindering interlayer mass transport. ${ }^{1,19}$ Our results demonstrate that the unstable interface evolution for $\mathrm{Cu}$ electrodeposition is enhanced by a nonuniform current distribution over the surface of the growing electrodeposit, as expected from contribution i. On the other hand, contributions ii-iv appear to be remarkably less important than contribution i. In fact, contribution iv can be disregarded, since for polycrystalline solids the existence of a Schwoebel-type energy barrier at step edges appears to be rather unlikely, ${ }^{20}$ contribution iii is not obvious in electrochemical solid-phase formations, and contribution ii results in growth patterns ${ }^{21}$ far from those found in our experiments.

The unstable interface evolution observed in additivefree plating baths is drastically changed when $\mathrm{Cu}$ electrodeposits are produced from thiourea-containing plating baths. Thus, for $r=90$ and $x=0.025$ (Figure 6a) only a

(17) Halpin-Healey, T.; Zhang, I. C. Phys. Rep. 1995, 254, 215.

(18) Amar, J.; Lam, P.-M.; Family, F. Phys. Rev. A. 1991, 43, 4548.

(19) Siegert, M.; Plischke, M. Phys. Rev. Lett. 1994, 73, 1517.

(20) Jeffries, J. H.; Zuo, J. K.; Creig, M. M. Phys. Rev. Lett. 1996, 76, 4931

(21) Albano, E.; Salvarezza, R. C.; Arvia, A. J. In preparation. 
a
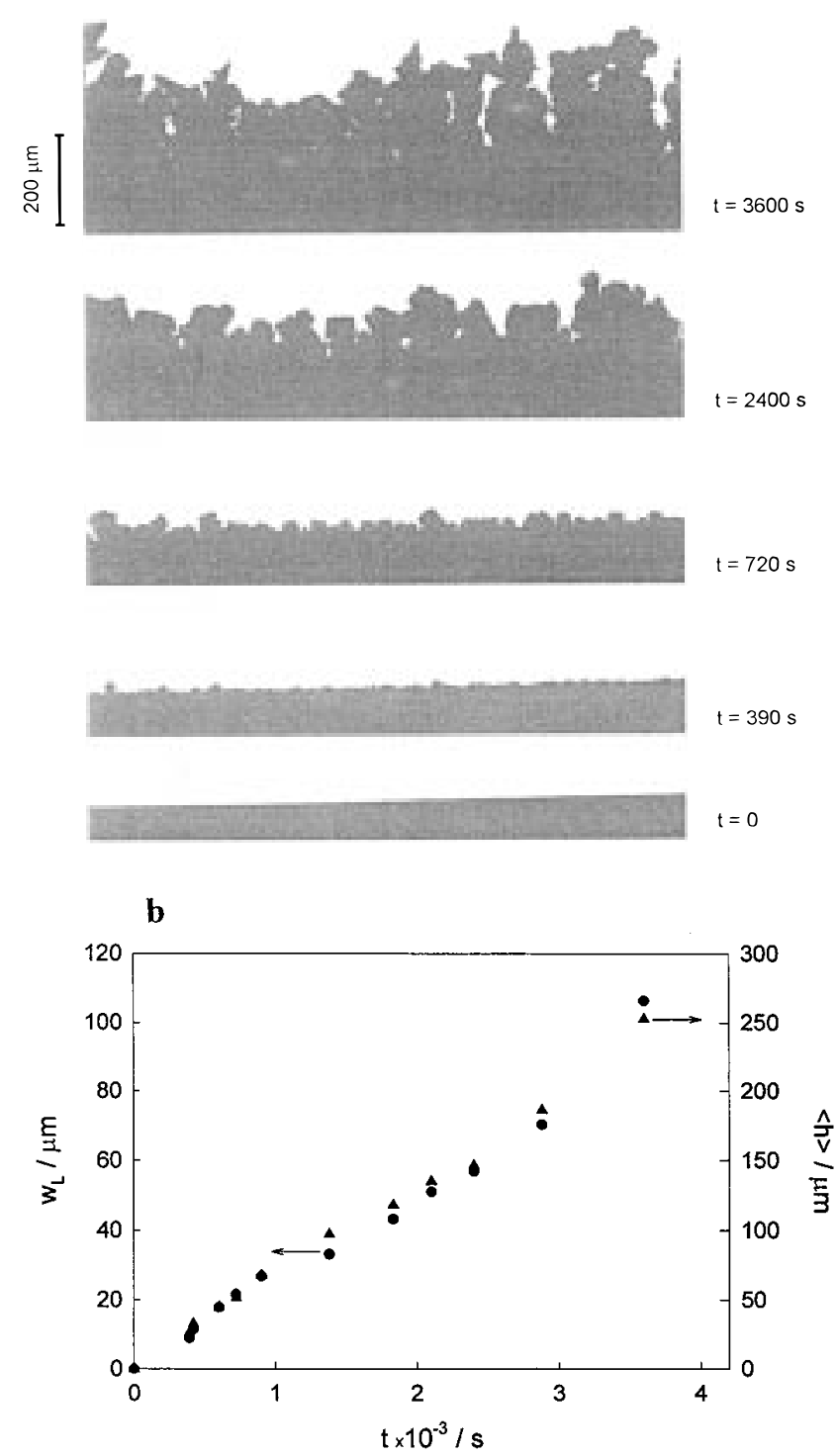

Figure 5. (a) In-situ micrographs showing the interface evolution for $\mathrm{Cu}$ electrodeposition from $0.6 \mathrm{M} \mathrm{CuSO}_{4} \cdot 5 \mathrm{H}_{2} \mathrm{O}+$ $0.5 \mathrm{M} \mathrm{H}_{2} \mathrm{SO}_{4}$ at $298 \mathrm{~K}$, using the $2 \mathrm{D}(r=37)$ cell. Gray shadowed portions correspond to the $\mathrm{Cu}$ phase. (b) $w_{\mathrm{L}}$ versus $t$ and $\langle h\rangle$ versus $t$ plots resulting from data taken from in-situ micrographs. The experimental conditions are the same as those for part (a).

few topographic features embedded into a nearly flat profile can be observed. The height of the deposit fulfills $\mathrm{a}\langle h\rangle \propto t$ relationship, but the value of $W_{\mathrm{L}}$ fluctuates around a low value (Figure $6 \mathrm{~b}$ ). In contrast to $\mathrm{Cu}$ electrodeposits grown in a thiourea-free plating bath where the unstable roughness growth regime could be followed for a long time, those electrodeposits produced in additive-containing plating baths for $x=0.025$ display small features having a lifetime of only about $60 \mathrm{~min}$. The evolution of one of these instabilities (Figure 7a) initially shows that the local rate of $\mathrm{Cu}$ electrodeposition there becomes greater than that at neighbor domains. Later on, the height of the nodule progressively decreases and it extends parallel to the substrate surface. Finally, the instability is completely suppressed. Then, for $x=0.025$ and a rather large electrodeposition time, the growth rate of $\mathrm{Cu}$ at nodules is substantially diminished in constrast to that of neighbor domains. The statistical birth and death of nodules a
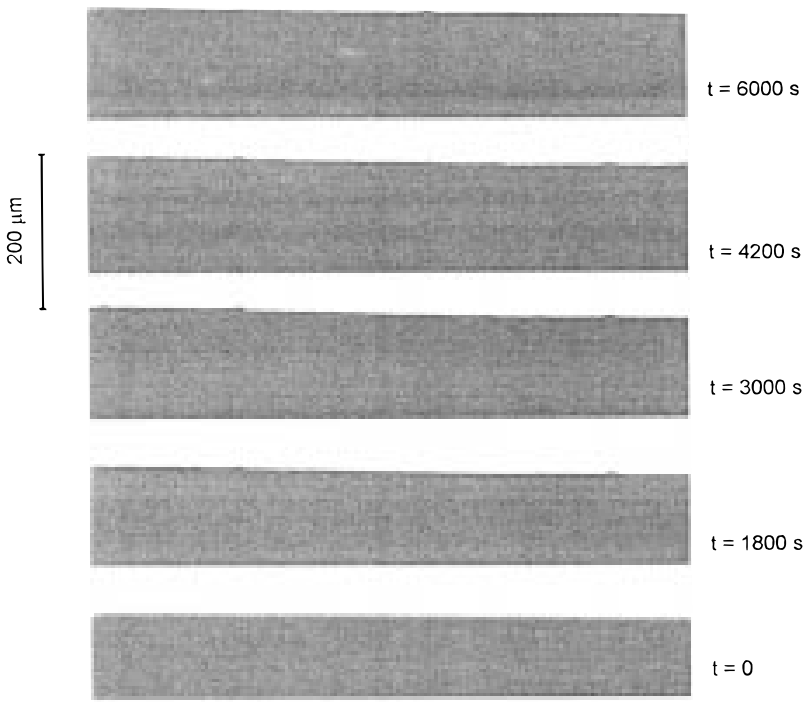

$\mathrm{b}$

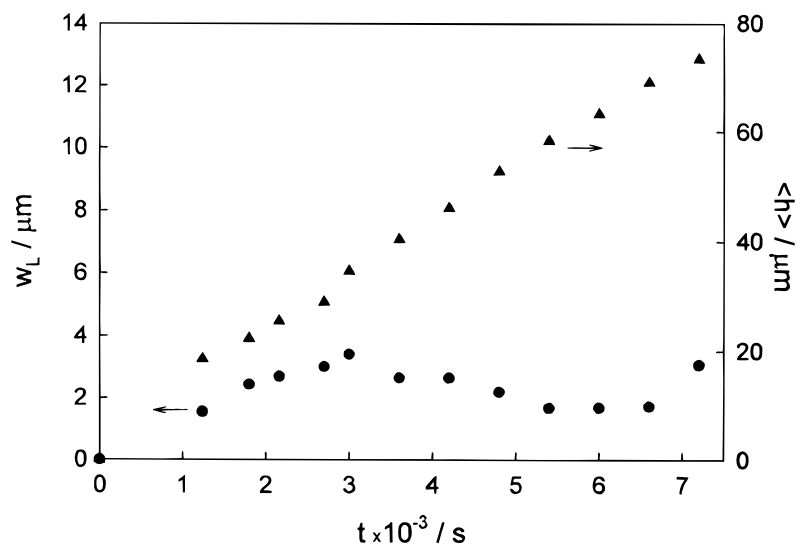

Figure 6. (a) In-situ micrographs showing the interface evolution for $\mathrm{Cu}$ electrodeposition from $0.6 \mathrm{M} \mathrm{CuSO}_{4} \cdot 5 \mathrm{H}_{2} \mathrm{O}+$ $0.5 \mathrm{M} \mathrm{H}_{2} \mathrm{SO}_{4}+0.025 \mathrm{M}$ thiourea at $298 \mathrm{~K}$, using the $2 \mathrm{D}(r=$ 37) cell. Gray shadowed portions correspond to the $\mathrm{Cu}$ phase. (b) $w_{\mathrm{L}}$ versus $t$ and $\langle h\rangle$ versus $t$ plots resulting from data taken from in-situ micrographs. The experimental conditions are the same as those for part (a).

produce fluctuations in the $W_{\mathrm{L}}$ versus $t$ plot around an average value of $W_{\mathrm{L}}$, and the instability width $(d)$ approaches a $d$ versus $t^{1 / z}$ relationship (Figure $7 \mathrm{~b}$ ) with $Z \cong$ 2 .

To explain the smoothing of the growth front, it might be assumed that the value of $K$ in the second term on the right hand side of eq 1 is increased, as would occur if the surface diffusion coefficient is increased by the presence of adsorbed thiourea. This situation, however, is opposite to that already reported for the influence of adsorbed organic molecules on surface atom diffusion on fcc metals, an example being the decrease by 2 orders of magnitude of the surface diffusion coefficient of $\mathrm{Au}$ adatoms on $\mathrm{Au}$ covered by alkanethiol molecules. ${ }^{22}$ Furthermore, for the above-described smoothing mechanism that operates at a much smaller scale length, the value $z=4$ should be expected. As this is not the case for our experiments at

(22) Chailapakul, O.; Sun, L.; Xu, C.; Crooks, R. M. J. Am. Chem. Soc. 1993, 115, 12459. 


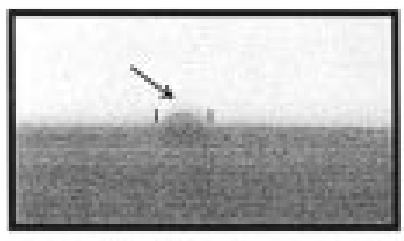

$t=1800 s$

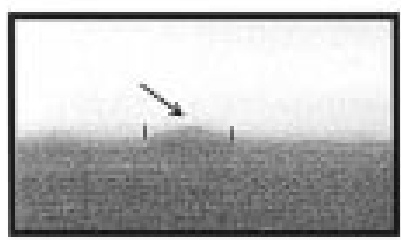

$t=4200 s$

b

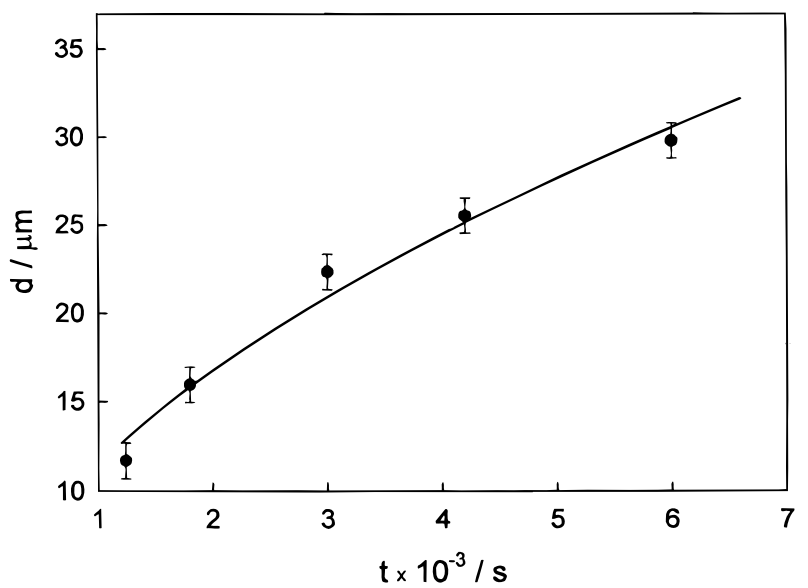

C

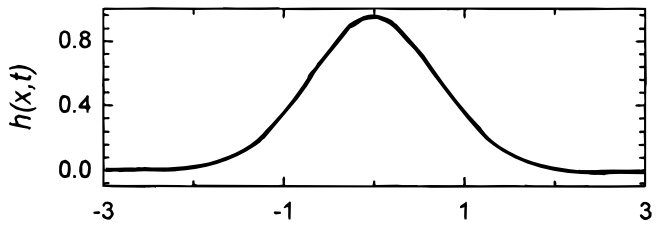

(I)

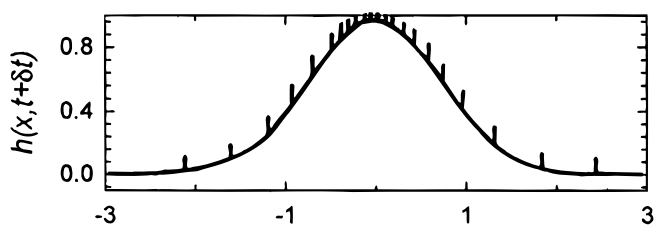

(II)
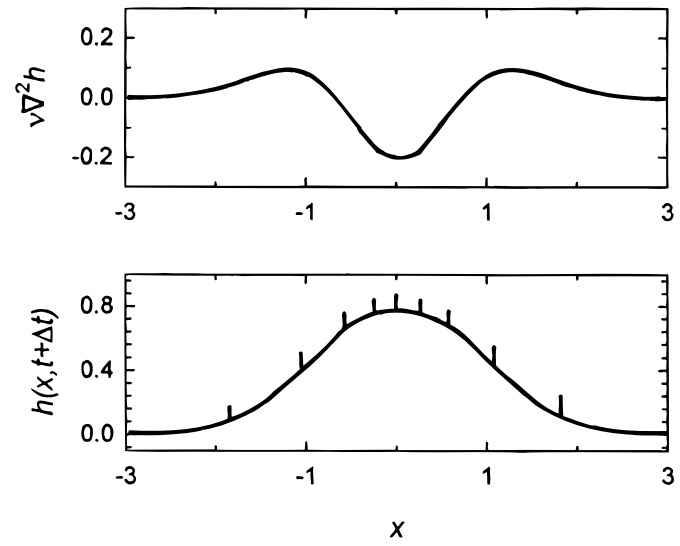

Figure 7. (a) In-situ evolution of a $\mathrm{Cu}$ protrusion formed at the interface. $\mathrm{Cu}$ electrodeposition conditions are indicated in Figure 6a. (b) $d$ versus $t$ plot resulting from data derived from part (a). (c) Effect of the surface coverage by the additive on the interface evolution. (I) At $t=0$, the random aggregation of particles $\eta(\mathbf{x}, t)$ in eq 1, produces a protrusion. (II) The adsorption of the additive occurs at $t+\delta t$. The density of small lines perpendicular to the $x$-axis at the interface represents the adsorbate distribution. (III) The term $v \nabla^{2} h$ in eq 1 gives the local acceleration of Cu electrodeposition around the protrusion. Minimum and maximum acceleration values are located at the protrusion top and valleys, respectively. (IV) As the protrusion height decreases, $\Delta t \gg \delta t$, the adsorbate density decreases. This diagram was adapted from ref 1.

relatively large scale lengths, a new explanation should be advanced to account for $z \cong 2$.

The value $z=2$ for a large scale length should be expected from the first term on the right hand side of eq $1,1,11$ that is, the EW equation. This equation predicts that the deposition rate is higher at valleys than at protrusions, resulting in the smoothening of the growth front. The underlying mechanism of this physical picture applied to the electrodeposition of $\mathrm{Cu}$ in the presence of thiourea implies the enhanced local adsorption of thiourea at protrusions, that is, at sites with a negative radius of curvature, ${ }^{23}$ as has been revealed by autoradiography and counting techniques. ${ }^{24}$ This preferential adsorption of thiourea would be favored by the higher electric field at those sites. ${ }^{25}$ Then, the local surface coverage by thiourea would hinder the electron-transfer reaction, as has recently been determined for a test redox reaction in

(23) Rudzinski, W.; Everett, D. H. Adsorption of Gases on Heteregeneous Surfaces; Academic Press: London, 1992; Chapter 2, p 148.

(24) Oniciu, L.; Muresan, L. J. Appl. Electrochem. 1991, 21, 565. Rogers, G. T.; Ware, M. J.; Fellows, R. V. J. Electrochem. Soc. 1960, 107, 677.

(25) Despic, A. R.; Popov, K. I. In Modern Aspects of Electrochemistry, Bockris, J. O'M., Conway, B. E., Eds.; Butterworths: New York, 1972 p 199. solution on thiol-covered Au electrodes. ${ }^{26}$ Accordingly, the presence of adsorbed thiourea on $\mathrm{Cu}$ protrusions would increase the local resistivity there, contributing to an increase in the local value of $\eta_{0}$ and a decrease in the local rate of $\mathrm{Cu}$ electrodeposition, compensated by an enhancement of $\mathrm{Cu}$ electrodeposition at valleys. From the crystallographic standpoint, it has been shown that in the presence of thiourea a preferred $\mathrm{Cu}(022)$ crystallographic orientation is produced, which is consistent with a ridge-type growth resulting in compact deposits. ${ }^{27}$ Consequently, the overall effect operating statistically over the entire surface of the electrodeposit is the hindrance of the vertical growth at protrusions and the favorable electrodeposition at valleys (Figure 7c) resulting from the $v \nabla^{2} h$ term in eq 1 . This situation leads to the development of a smooth $\mathrm{Cu}$ electrodeposit. Then, the presence of thiourea for $x>0.025$ promotes a leveling effect. The existence of a critical concentration of thiorea would be related to a minimum value of $\theta$ at protrusions required to decrease the $\mathrm{Cu}$ electrodeposition rate to a value lower than that operating at valleys and flat portions of the

(26) Sabatini, E.; Rubinstein, I. J. Phys. Chem. 1987, 91, 6663.

(27) Lakshmanan, V. I.; Mackinnon, D. J.; Brannen, J. M. J. Appl. Electrochim. 1977, 7, 127. 
electrode surface. As the radius of curvature at surface domains decreases due to the lateral growth, adsorbed molecules either desorb or diffuse on the surface toward neighbor irregularities where their adsorption is favored. The difference in the chemical potential for thiourea adsorption at valleys and protrusions constitutes the driving force for the displacement of thiourea molecules over the growing $\mathrm{Cu}$ electrodeposit.

In conclusion, the overall influence of thiourea on $\mathrm{Cu}$ electrodeposition is to slow the growth rate at negatively curved surface domains (protrusions). The reverse effect operates at flat surface domains or valleys. Then, the dynamics of the $\mathrm{Cu}$ /solution smooth interface motion at the millimeter scale length can be satisfactorily described by the EW equation.

Acknowledgment. This work has been supported by Grants PIA 7283/97 and PIP 014/97 from CONICET (Argentina), Projects 2950054 and 197244 from FONDECYT (Chile), and the Scientific Cooperation Program supported by Fundación Andes (Chile) and Fundación Antorchas (Argentina).

LA971358K 